WellBeing International

WBI Studies Repository

$9-2000$

\title{
Diversity of Behaviour During Novel Object Tests is Reduced in Pigs Housed in Substrate-Impoverished Conditions
}

\author{
Françoise Wemelsfelder \\ Scottish Agricultural College \\ Marie Haskell \\ The Roslin Institute \\ Michael T. Mendl \\ University of Bristol \\ Sheena Calvert \\ Scottish Agricultural College \\ Alistair B. Lawrence \\ Scottish Agricultural College
}

Follow this and additional works at: https://www.wellbeingintlstudiesrepository.org/acwp_asie

Part of the Agribusiness Commons, Animal Studies Commons, and the Operations and Supply Chain Management Commons

\section{Recommended Citation}

Wemelsfelder, F., Haskell, M., Mendl, M. T., Calvert, S., \& Lawrence, A. B. (2000). Diversity of behaviour during novel object tests is reduced in pigs housed in substrate-impoverished conditions. Animal Behaviour, 60(3), 385-394.

This material is brought to you for free and open access by WellBeing International. It has been accepted for inclusion by an authorized administrator of the WBI Studies Repository. For more information, please contact wbisr-info@wellbeingintl.org.

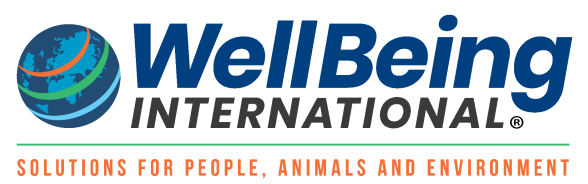




\title{
Diversity of Behaviour During Novel Object Tests is Reduced in Pigs Housed in Substrate- Impoverished Conditions
}

\author{
Françoise Wemelsfelder ${ }^{1}$, Marie Haskell ${ }^{2}$, Michael T. Mendl ${ }^{3}$, Sheena Calvert ${ }^{1}$, and Alistair B. Lawrence ${ }^{1}$ \\ ${ }^{1}$ Scottish Agricultural College \\ ${ }^{2}$ The Roslin Institute \\ ${ }^{3}$ University of Bristol
}

\begin{abstract}
Modern intensive farming conditions lack the diversity of substrates present in more natural environments and offer young animals fewer opportunities for interaction. Evidence exists that this may affect the organization of interactive patterns of behaviour, but shifts in behavioural diversity have not been measured directly. We investigated the effect of the substrate in the home pen on the diversity of behaviour in young growing pigs, Sus scrofa. Over 5 months, 26 pigs were housed singly in either substrate-impoverished (SI) or substrate-enriched (SE) conditions. Once every month we recorded the behaviour of these pigs in detail both in the home pens and in two novel object tests. In addition, we calculated the diversity of behaviour shown by SI and SE pigs in the home pen and in the novel object tests, using a relative behavioural diversity index. In the two novel object tests, SI pigs were less mobile than SE pigs and focused their behaviour on particular substrates. In addition, SI pigs showed less diverse behaviour than SE pigs. Our results show that the less diverse behaviour of SI pigs previously recorded in their home pens persists under novel conditions, supporting the hypothesis that substrateimpoverished housing conditions structurally affect the organization of behaviour in young growing pigs.
\end{abstract}

In modern intensive farming systems, young animals are often housed in conditions that lack the diversity of stimulation present in more natural environments (Wood-Gush \& Vestergaard 1989). First, this may affect the expression of individual elements of behaviour. Social and appetitive behaviours may decrease in frequency, while various redirected behaviours may emerge (McKinnon et al. 1989; Petersen et al. 1995). Second, shifts in interactive patterns of behaviour have been observed. Animals may be less able to engage in complex interaction with novel objects (Renner 1987) or to inhibit or reverse previously acquired response patterns (Morgan 1973; Einon et al. 1978; however, this effect could not be reproduced with pigs, Sus scrofa: Mendl et al. 1997) and their behaviour may become less diverse and/or more stereotyped (Stolba et al. 1983; Dantzer 1986; Mason 1991; Gunn \& Morton 1995). The majority of studies have investigated such shifts in interaction in terms of specific behavioural categories such as play, various forms of operant behaviour, or stereotyped behaviour sequences (e.g. Morgan 1973; Einon et al. 1978; Page \& Neuringer 1985; Dantzer 1986). In other cases, several classes of behaviour are grouped into one category which is then taken as an index of behavioural diversity (e.g. Renner \& Rosenzweig 1986; Gunn \& Morton 1995). Few studies, however, have attempted to measure shifts in the diversity of behaviour more directly, by detailed investigation of the interactive patterns constituting the 
whole behavioural repertoire. Such investigation could shed light on the role of behavioural diversity in proximate, causal models of behavioural ontogeny and development.

We investigated the effect of the substrate in the home pen on the diversity of the interactive repertoire of young growing pigs. In a previous paper (Haskell et al. 1996) we reported that the behaviour of pigs is less diverse in substrate-impoverished housing conditions (SI) than in substrate-enriched conditions (SE). To measure behavioural diversity, we used a relative diversity parameter, $R$, which takes into account the fewer opportunities to interact with suitable substrate in improverished than in enriched conditions. $R$ represents the number of behavioural elements observed as a proportion of the total repertoire possible in that environment (cf. Losey 1978; Stolba et al. 1983). In Haskell et al. (1996), we suggested that the reduced relative behavioural diversity of SI pigs may either be specific to the home pen situation, reflecting their low motivation to interact with unsuitable substrates, or reflect a more pervasive shift in the organization of their behaviour.

To test which of these hypotheses may be correct, we considered the relative behavioural diversity of SI and SE pigs (the same as in the home pen study) under novel environmental conditions. Novelty tests are widely used to investigate changes in the organization of behaviour; however, their interpretation depends on how the novel situation is presented to the animal. Welker (1957) distinguished between 'free' and 'forced' exploration, arguing that the two situations evoke different types of response. The majority of novelty tests, including open field tests, should in this light be regarded as 'forced' in nature, in that they remove or isolate animals from their home environment (e.g. Meijsser et al. 1989; Pearce \& Paterson 1993; Prior \& Sachzer 1994; de Passillé et al. 1995). Authors may claim that exploration of the open field is 'free' because of the absence of specific operant tasks (Belzung \& Le Pape 1994), or because the animal can choose not to emerge from a starting box (Welker 1957; Renner \& Rosenzweig 1986), but the fact remains that animals do not face the novel situation by choice. Open field tests are therefore primarily suited to studies of fear and anxiety investigating an animal's response to novel challenges (Treit 1985; Belzung \& Le Pape 1994; Boissy \& Bouissou 1995).

If an animal's propensity to interact with the environment is to be investigated in its own right, disentangled from the animal's response to a fear-inducing situation, the exploration needs to be truly 'free'. The few studies meeting this criterion found young piglets to be highly responsive to such conditions (Wood-Gush et al. 1990; Wood-Gush \& Vestergaard 1991). However, in these studies only a few specific variables, such as latency of approach and time spent interacting with the novel object, were measured and the issue of behavioural diversity was not addressed. We therefore investigated patterns of interaction and their diversity under conditions of voluntary exploration, using the same measurement technique as described in Haskell et al. (1996). Our null hypothesis was that the behavioural diversity of SI and SE pigs in the novelty tests would be similar indicating that the reduced behavioural diversity in SI pigs reported by Haskell et al. (1996) was specific to the home pen situation. Falsification of the null hypothesis, however, would indicate a more general shift in the behavioural organization of SI pigs.

\section{METHODS}

Animals and Housing

We used 26 Large White $\times$ Landrace female pigs, ca. 10 weeks of age and weighing between 28.0 and $45.0 \mathrm{~kg}$ (mean $37.1 \mathrm{~kg}$ ) at the beginning of the experiment. All pigs were housed within the same experimental house, in two experimental rooms, identical in construction, heating and ventilation, and maintained at $17-20^{\circ} \mathrm{C}$ throughout the experiment. Each pig was housed individually in a pen of $2 \times 3 \mathrm{~m}$. Individual housing was necessary to avoid confounding the effects of environmental and social 
enrichment on behaviour. However, the pen sides were barred, so that pigs in the same treatment could have visual contact and snout contact with neighbours.

We created two treatments by dividing the pigs into two groups of 13 , balanced for weight. One group was housed in substrate-impoverished $(\mathrm{SI})$ pens with bare concrete floors. The other was housed in substrate-enriched (SE) pens containing ca. $4 \mathrm{~kg}$ of straw, $5 \mathrm{~kg}$ of forest bark and two tree branches. In each experimental room, a row of substrate-impoverished pens lay back-to-back with a row of substrateenriched pens, eight pairs in one room and five in the other. Pigs had snout and visual contact with animals in their treatment group, but not with those in the other treatment group. All pens contained a drinker bowl and a food trough, and the pigs had an ad libitum supply of water and food appropriate to their age. Food (Growlean E. R. Pellets, BOCM Pauls Ltd, Ipswich, U.K.) was provided at 0830 hours each day and pens were cleaned at ca. 0900 hours. The bare concrete floors were scraped clean, and soiled straw and bark were removed and replaced with fresh material. Tree branches were changed once a month.

\section{Experimental Procedures}

We presented pigs in both treatments with two types of 'novel object test' once a month over 5 months, starting 1 month after the pigs had entered their treatment conditions. These tests were the 'arena novel object test' and the 'home pen novel object test'. Testing took place on 4 consecutive days each month, the first 3 days for the arena test (10 pigs on days 1 and 2 and 6 on day 3 ) and the fourth for the home pen test (all 26 pigs). The order (day and time of day) in which individual pigs were tested was balanced over the months by a Latin square design. We selected 10 novel objects, five for each test, for presentation to the pigs. These objects were artificial, to avoid confounding them with the substrateenriched pigs' greater experience with natural substrates. In the arena novel object test, we used a plastic toy tractor, a rubber bicycle tyre, a set of plastic wheels, a small plastic buggy and a plastic soap tray, whereas in the home pen test we used a plastic watering can, a plastic bucket, a rubber wellington boot, a three-legged wooden stool and a car steering wheel in rubber coating. These objects are of considerable interest to pigs ( $R$. Kirkden, unpublished data). Over the 5-month experimental period, each pig was presented once with each object in each test. The order in which objects were presented to each pig was balanced using a Latin square design.

In the arena novel object test, we placed the novel object in an arena of $1 \times 3 \mathrm{~m}$ that consisted of six solid metal panels and that could be moved along the corridor to be attached to each focal pig's home pen. Testing took place in two consecutive trials, each 10 min long. Each trial started when we opened the gate of the home pen, giving pigs the opportunity to enter the arena. The first trial, called 'habituation', allowed pigs to familiarize themselves with the arena without a novel object present. After 10 min we guided the pigs back to their home pen, shut the gate, and placed a novel object at the far end of the arena. The gate was then opened again and pigs given a further $10 \mathrm{~min}$ to explore the arena and the object. In the home pen novel object test, we placed the novel object directly into the animal's home pen. Testing in this case lasted $10 \mathrm{~min}$, as there was no habituation period.

We recorded the behaviour of pigs continuously with the Keytime program (Deag 1993). The ethogram for animals in both treatments included location (home pen or arena), posture (stand, sit, lie on belly, lie on side), behaviour pattern (nose, chew, root, lick, carry, shake, pull, rub, drink, eat, scamper, orient to observer, paw, tailwag, stand or walk idle, change area) and substrate at which any one behaviour pattern was directed (novel object, arena sides, floor, drain, bars, food trough, neighbouring pig, loose substrate). Full details of this ethogram are given in Haskell et al. (1996). The observer was seated behind a wooden screen, which in the arena test was placed at the end of the arena opposite to the novel 
object, and in the home pen test in front of the focal animal's pen. If at the beginning of a test period a pig was not standing, we gently induced it to stand.

\section{Statistical Analysis}

To process the data, we used Keytime (Deag 1993) to produce the mean frequency, mean duration and mean bout length of behavioural elements during the 10-min trials. As there were many zero values in the data, which increased the level of non-normality, we grouped individual elements of behaviour into larger 'substrate categories' (i.e. all behaviour directed at, for example, novel object, arena floor or home pen bars). However, interactions with the novel object were also analysed in terms of their individual elements of behaviour.

To increase the normality of the distribution of the data, we used a square root transformation for statistical analysis. Since each month five objects had to be presented to 26 pigs, the experimental design of the novel object tests could not be fully balanced. We therefore used REML (REsidual Maximum Likelihood) to test the effect of treatment and month on the occurrence of behaviours (Patterson \& Thompson 1971). Treatment, month and time of day were used as fixed effects and pig identity was fitted as a random effect.

REML uses Wald statistics to test the significance of fixed effects as they are added to the model (Lawes Agricultural Trust, Genstat 5 Manual 1993). The Wald statistic has an asymptotic chi-square distribution with degrees of freedom equal to those of the terms in the fixed model. Since most of the variables were not normally distributed, significance levels for the Wald statistics were obtained by a randomization method (Adams \& Anthony 1996). In this method repeated samples were taken from the data, randomly assigned to the treatments and the REML analysis rerun. This technique allows the null distribution of the relevant Wald test statistics to be obtained and hence enables a $P$ value to be assigned to the actual values obtained. Wald statistics $(W)$ for month effects and month-by-treatment interactions in the Results are based on $d f=4$; those for treatment effects, in Tables 3 and 4 in the Results, are based on $d f=1$. To analyse the effect of treatment and month on the bout length of various behaviours, we restricted the data set to exclude zero values as we wished only to include data sets in which bouts of relevant behaviour had actually occurred.

Since novel objects were absent in the habituation trials, the problem of unbalanced design did not arise. In these trials, we therefore determined the effect of treatment and month on the behaviours that achieved normality by parametric ANOVA. The analysis was blocked for the between-cell effects of pig within pair, time of day within month and observer. $F$ values for month effects and month-by-treatment interactions in the Results are based on $d f=4$; those for treatment effects, in Table 2 in the Results, are based on $d f=1$.

To examine the diversity of behaviour, we calculated an index of both the absolute diversity $(H)$ and the relative diversity $(R)$ for each treatment (Losey 1978; Stolba et al. 1983). $H$ provides a measure of diversity within a given sequence of behavioural flow. This measure is dependent both on the number of behavioural elements in that sequence (e.g. three elements: nose bar, lick floor, chew straw) and on the distribution of the frequencies with which those elements are initiated (e.g. nose bar 10x, lick floor $3 \times$, chew straw $8 \times$ ). The higher the number of elements and the more equally distributed their frequencies, the higher the value of $H . R$ is then calculated by dividing $H$ by $H_{\max } . H_{\max }$ represents the maximum absolute diversity possible within a given treatment condition (i.e. all elements of the ethogram are observed and occur equally often; for example nose bar $7 \times$, lick floor $7 \times$, chew straw $7 \times$ ). $H_{\max }$ will be lower in substrate-impoverished (SI) conditions than in substrate-enriched (SE) conditions, because there are fewer substrates available for interaction (e.g. chew straw is not available to SI pigs). Thus, $R$ is a 
relative value, reflecting the diversity of behaviour in a given environment $(H)$ as a function of the behavioural options available in that environment $\left(H_{\max }\right)$.

We calculated two types of $R$ value. One type concerned the total interactive repertoire and used all behaviours in combination with all substrates as elements in the analysis. The other type concerned manipulative behaviours only, and included nose, chew, root, carry/shake, lick and paw in combination with all substrates. We calculated $R$ for each pig at each monthly observation point in each of the three experimental trials: the arena habituation trial, the arena novel object trial and the home pen novel object trial. These values were checked for normality of distribution and analysed for the effects of treatment and month by ANOVA ( $F$ values, in Table 1 in the Results, are based on $d f=1$ ).

The calculation of $R$ is generally sensitive to the 'grain' of the ethogram used (i.e. its number of behavioural categories), and so whether significant differences in $R$ between $\mathrm{SI}$ and SE pigs are found could be biased by the classification system. We used a fine-grained ethogram in that it contained all possible combinations of behavior and substrate (e.g. chew branch, carry straw). From this ethogram SE pigs had a much larger set of possible interactive patterns at their disposal than SI pigs, and so to obtain the same relative diversity scores as SI pigs they had to engage in considerably more behaviours than SI pigs. Thus even though $R$ values are to some extent sensitive to methodological bias, this is not likely to lead to unjustified support for the ideas that underlie this research.

Table 1. Effect of treatment on the relative diversity of behaviour of pigs housed in substrate-enriched (SE) or substrate-impoverished (SI) conditions

\begin{tabular}{|c|c|c|c|}
\hline & \multicolumn{2}{|c|}{ Relative diversity $(R)$} & \multirow[b]{2}{*}{$F_{1,12}$} \\
\hline & SE & SI & \\
\hline \multicolumn{4}{|l|}{ All behaviours } \\
\hline \multicolumn{4}{|l|}{ Arena test } \\
\hline Habituation trial & $0.67 \pm 0.03$ & $0.61 \pm 0.05$ & $8.34^{\star \star}$ \\
\hline Novel object trial & $0.55 \pm 0.03$ & $0.48 \pm 0.03$ & $12.42^{\star *}$ \\
\hline Home pen test & $0.49 \pm 0.02$ & $0.45 \pm 0.02$ & $7.65^{\star}$ \\
\hline \multicolumn{4}{|c|}{ Manipulative behaviours } \\
\hline \multicolumn{4}{|l|}{ Arena test } \\
\hline Habituation trial & $0.65 \pm 0.03$ & $0.60 \pm 0.05$ & $5.01^{*}$ \\
\hline Novel object trial & $0.55 \pm 0.03$ & $0.46 \pm 0.04$ & $12.47^{* *}$ \\
\hline Home pen test & $0.48 \pm 0.02$ & $0.44 \pm 0.02$ & $6.34^{*}$ \\
\hline
\end{tabular}

Means are given \pm SEM.

${ }^{\star} P<0.05 ;{ }^{\star \star} P<0.01$.

\section{RESULTS AND DISCUSSION}

\section{Relative Behavioural Diversity}

In all three trials, the arena habituation trial, the arena novel object trial and the home pen novel object trial, the behaviour of SI pigs was significantly less diverse than that of SE pigs (Table 1). There was no effect of month on either value of $R$ in any of the tests. 


\section{Arena Habituation Trial}

A significant interaction between month and treatment effects occurred with respect to frequency and duration of scampering (frequency: $F_{4,43}=2.96, P<0.05$; duration: $F_{4,43}=3.64, P<0.05$; Fig. 1a), suggesting that the SI treatment had a restraining effect on the development of play. Furthermore, the latency to enter the test arena decreased for all pigs as the experiment progressed $\left(F_{4,48}=17.36, P<\right.$ 0.001 ; Fig. $2 a$ ), suggesting a gradual reduction in fear.

The behaviour of SE and SI pigs during this trial differed significantly in several ways (Table 2). First, SE pigs were more mobile than SI pigs: they went back and forth between home pen and arena more frequently, scampered more frequently and for longer for the first 3 months (see above), and also stood or walked without attending to any substrate more frequently and for longer. Second, they interacted more with a variety of substrates than SI pigs: they made contact with their neighbours more often and for longer, tended to interact more often with the side panels of the arena, although not significantly so, and interacted more with loose substrate. They also drank more often and for longer and pawed and wagged their tails more often.

SI pigs interacted with the floor more frequently, and for almost twice as long as SE animals, and did so with a significantly longer bout length (SI pigs: $\bar{X} \pm$ SEM $=9.81 \pm 1.49 \mathrm{~s}$; SE pigs: $6.11 \pm 0.65 \mathrm{~s} ; F_{1,12}=$ $16.74, P<0.001)$. This difference was mostly due to floor-nosing behaviour, which showed a significant difference in frequency and duration (Table 2) and bout length (SI pigs: $\bar{X} \pm$ SEM $=7.83 \pm 0.88 \mathrm{~S}$; SE pigs: $\left.5.38 \pm 0.50 \mathrm{~s} ; F_{1,12}=14.65, P<0.01\right)$, and to a lesser extent to floor licking, which showed a significant difference in frequency and duration.

\section{Arena Novel Object Trial}

Several interactions of month and treatment effects were found in the arena novel object trial. First, SI pigs took longer to enter the arena in the first month, but did so faster than SE pigs at month $5\left(W_{4}=9.4\right.$, $P<0.05$; Fig. 1b). SI animals also took longer to start interacting with the novel object in the first month, coming down to the level of SE animals by month $3\left(W_{4}=11.7, P<0.05\right.$; Fig. $\left.1 c\right)$. This suggests that at the start of the experiment SI pigs were more fearful of approaching the novel object than SE pigs. Second, SI animals showed a steep increase in time spent chewing the novel object from month 3 onwards, an effect not shown by SE pigs (frequency: $W_{4}=11.1, P<0.05$; duration: $W_{4}=11.3, P<0.05$; Fig. 1d).

The behaviour of all pigs in the arena novel object trial showed various effects of month. As the experiment progressed, pigs oriented less towards the novel object (frequency: $W_{4}=19.3, P<0.001$; duration: $W_{4}=18.5, P<0.001$; Fig. $2 \mathrm{~b}$; bout length: $\left.W_{4}=11.0, P<0.05\right)$. Interaction with the novel object generally increased as the experiment progressed (frequency: $W_{4}=25.6, P<0.001$; duration: $W_{4}=32.2$, $P<0.001$; Fig. 2c; bout length: $W_{4}=9.8, P<0.05$ ), owing mainly to an increase in nosing the object by all animals (frequency: $W_{4}=42.2, P<0.001$; duration: $W_{4}=24.9, P<0.001$; Fig. $2 \mathrm{c}$ ) and in chewing the object by SI pigs (frequency: $W_{4}=49.5, P<0.001$; duration: $W_{4}=36.2, P<0.001$; Fig. $2 c$; see also Fig. 1d). These effects suggest that as the experiment progressed pigs became less fearful of approaching the novel object.

SE and SI pigs differed in their behaviour during this trial in several ways (Table 3), most of which were similar to those observed in the habituation trial. SE pigs were more mobile than SI pigs: they went back and forth between home pen and arena more frequently, scampered more frequently and for longer, and stood or walked without attending to any substrate more frequently. They also interacted more with a variety of substrates, interacting more with the drain, arena side panels, bars and loose substrates, and 
making contact with their neighbours more frequently. SE pigs furthermore wagged their tails more often, scratched themselves, more and for longer, and drank and defecated more.

SI pigs differed from SE pigs in that from month 3 onward they chewed the novel object for longer (see Fig. 1d) with a significantly longer bout length (SI pigs: $\bar{X} \pm$ SEM $=7.12 \pm 0.25$ s; SE pigs: $5.30 \pm 0.22 \mathrm{~s}$; $\left.W_{1}=27.6, P<0.001\right)$.

Figure 1. Interactions between the effect of month and treatment on the duration or latency (s) of behaviours in a 10-min test for pigs housed in substrate-enriched $(\bullet)$ or substrate-impoverished $(\circ)$ conditions. Means are given \pm SEM. (a) Arena habituation trial; (b-d) arena novel object trial; (e, f) home pen novel object trial.
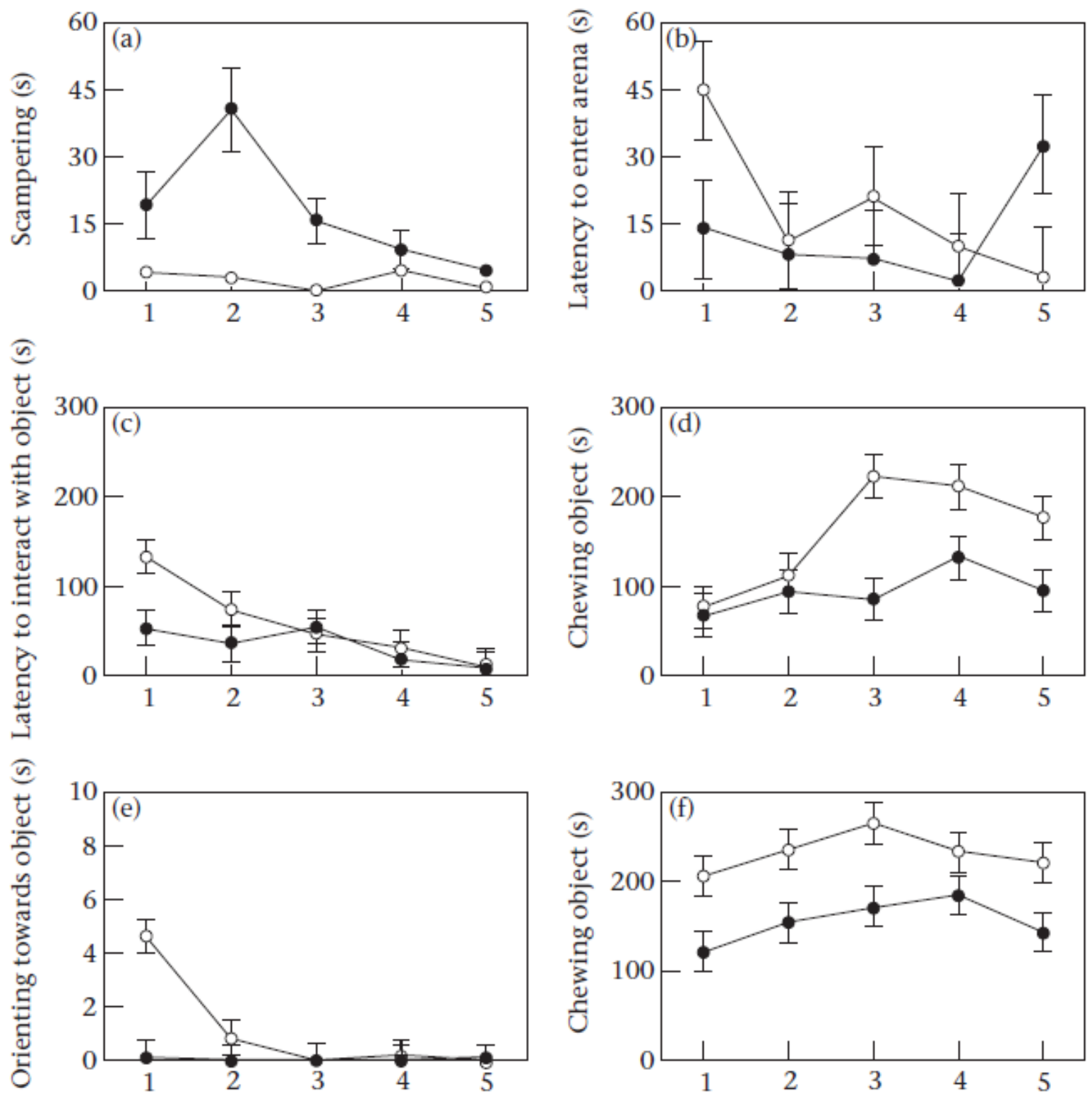

Month 


\section{Home Pen Novel Object Trial}

In this trial one significant interaction between month and treatment effects occurred. At the start of the experiment SI pigs oriented towards the object more than SE pigs, but came down to the level of SE animals by month 3 (frequency: $W_{4}=14.3, P<0.01$; duration: $W_{4}=19.3, P<0.001$; Fig. 1 e). This suggests that, as in the arena trial, SI pigs in the home pen trial were initially more fearful of approaching the novel object than SE pigs.

Figure 2. The effect of month on the latency (a), duration (b-d) or bout length (e) (s) of behaviours of all pigs in a 10-min test. Means are given \pm SEM. (a) Arena habituation trial; (b, c) arena novel object trial; (d, e) home pen novel object trial. In (c): •: interacting with object; $\mathbf{n}$ : chewing object; $\boldsymbol{\Delta}$ : nosing object.
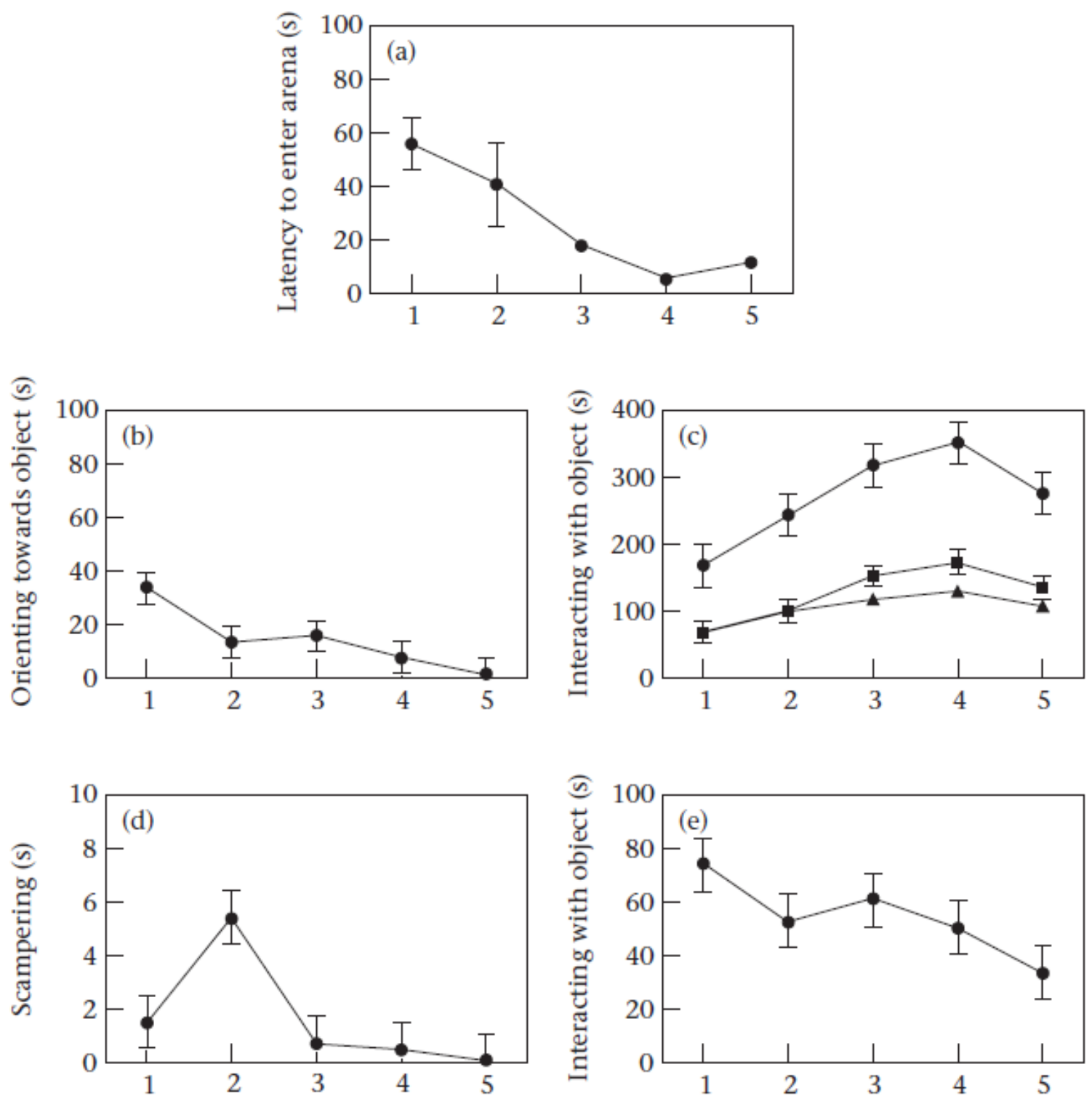

Month 
Various effects of month were also found. Scampering showed a peak at month 2 (frequency: $W_{4}=28.4$, $P<0.001$; duration: $W_{4}=34.8, P<0.001$; Fig. 2 d), which concurs with the high level of scampering noted during the home pen observations at that time (Haskell et al. 1996). Similar to the arena novel object trial, interaction with the novel object increased (frequency: $W_{4}=26.4, P<0.001$ ), indicating declining fear. In contrast to the arena trial, however, mean bout length of overall interaction with the object declined over time $\left(W_{4}=16.8, P<0.01 ;\right.$ Fig. $\left.2 \mathrm{e}\right)$.

The behaviour of SE and SI pigs showed several significant differences in this trial (Table 4). Similar to both the arena habituation and novel object trial, SE pigs were more mobile and interacted more with a variety of substrates. They stood or walked without attending to any substrate more often, interacted more frequently with the bars of the pen and with neighbouring pigs, and also drank more. Furthermore, SE pigs looked at observers more frequently and interacted more with loose substrate in the pen, while SI animals interacted more with the pen floor.

SI pigs took more time to start interacting with the object (SI pigs: $\bar{X} \pm$ SEM $=4.49 \pm 0.55$ s; SE pigs: 2.17 $\left.\pm 0.55 \mathrm{~s} ; W_{1}=8.2, P<0.01\right)$. Once started, SI animals interacted with the novel object for substantially longer than SE animals, nosing it more frequently and, as in the arena test, chewing it more and for longer (Fig. 1f) in longer bouts (SI pigs: $\bar{X} \pm$ SEM $=6.40 \pm 0.26 \mathrm{~s}$; SE pigs: $5.11 \pm 0.26 \mathrm{~s} ; W_{1}=14.6, P<$ $0.001)$. SE pigs by contrast rooted the object more often and for longer.

\section{GENERAL DISCUSSION}

Haskell et al. (1996) found that pigs housed in substrate-impoverished conditions had less diverse behaviour than pigs housed in substrate-enriched conditions. Our results show that this effect is not limited to the home pen situation. In both novel object tests, each taken once a month over a 5-month period, substrate-impoverished (SI) pigs had less diverse behaviour than substrate-enriched (SE) pigs. Since our index of diversity, $R$, depends on the number of elements used in a behaviour sequence and on the distribution of the frequencies of those elements (see Methods), this result indicates that SI pigs consistently used fewer elements in their behavioural interactions with the two novel object tests.

This difference in measured behavioural diversity is consistent with the other significant behavioural differences between SE and SI pigs. SE pigs were more mobile (walking, scampering) than SI pigs and interacted more frequently with a variety of substrates. They also showed higher levels of other behaviour which may indicate heightened excitement (drinking, and in some tests also pawing, tail wagging, scratching, defecation and looking at the observer). SI pigs were initially more fearful of the novel object and chewed it for significantly longer with significantly longer bout lengths than SE animals. During the arena habituation trial when the novel object was absent, SI pigs nosed the floor significantly more and with significantly longer bout lengths than SE pigs.

Thus the higher levels of fear behaviour, object chewing and floor nosing in SI pigs apparently occured at the expense of the behavioural mobility, variety and excitement shown by SE pigs. This shift in behaviour of SI pigs could simply be a matter of replacement, where one behaviour substitutes other less urgent behaviour. The SI pigs' motivation to chew and nose was likely to be thwarted in the home pen by the lack of suitable substrates, and in the presence of novel stimulation may have acquired a high priority and resulted in rebound chewing/nosing activity. The higher bout length of both these activities in SI pigs particularly suggests a difference in underlying motivation with SE pigs (Hughes \& Duncan 1988).

However, assuming a linear causal link between levels of chewing/nosing and reduced behavioural diversity may be too simple. SI and SE pigs did not just do different things; they differed in the type of interaction. Behaviour characterized by mobility, variety and excitement in SE pigs was reduced in favour 
of behaviour focused on particular substrates in SI pigs. This effect persisted over 5 months in all tests whether or not the novel object was present. Although some of the differences in behaviour were small (e.g. scampering), we suggest that qualitatively they may be important. Against the background of the reduced $R$ value in SI pigs, their greater focus on particular substrates may indicate an underlying shift towards a more restricted, less flexible organization of behavior (Wemelsfelder 1993). This interpretation is still an hypothesis and should stimulate further investigation. Future research should continue to measure, as we did in this study, both specific behavioural elements and general behavioural diversity. The latter measure is based on the combined frequency distributions of all behavioural elements, addressing behavioural diversity more directly and inclusively than the measurement of specific elements ever could. Specific measurements, on the other hand, may clarify the mechanisms underlying shifts in general behavioural diversity. The question is whether, and how, the two types of measurement covary in response to the experimental manipulation of relevant behavioural variables (e.g. chewing). One could, for example, provide SI pigs with different opportunities for specific behaviour (e.g. something to chew or nose in an otherwise impoverished home pen environment), and again investigate their response to the novel object tests. If a significant reduction in behavioural diversity were persistently found regardless of any specific effect that may arise, then the hypothesis that under SI conditions a nonspecific shift in behavioural organization may occur would gain increasing support.

Table 2. Effect of treatment on the behaviour of pigs housed in substrate-enriched (SE) or substrateimpoverished $(\mathrm{SI})$ conditions in the arena habituation trial

\begin{tabular}{|c|c|c|c|c|c|c|}
\hline \multirow[b]{2}{*}{ Behaviour } & \multicolumn{2}{|c|}{ Frequency } & \multirow[b]{2}{*}{$F_{1,12}$} & \multicolumn{2}{|c|}{ Duration } & \multirow[b]{2}{*}{$F_{1,12}$} \\
\hline & SE & SI & & SE & SI & \\
\hline Change area & $8.0 \pm 1.1$ & $3.5 \pm 0.6$ & $21.3^{\star * \star}$ & --- & --- & \\
\hline Stand/walk idle & $34.5 \pm 3.0$ & $26.3 \pm 2.9$ & $12.2^{\star \star \star}$ & $139.9 \pm 18.0$ & $100.7 \pm 14.8$ & $10.7^{\star \star}$ \\
\hline Scamper & $4.4 \pm 1.4$ & $1.1 \pm 0.5$ & $24.6^{\star \star \star}$ & $17.7 \pm 6.7$ & $2.4 \pm 2.3$ & $19.8^{\star \star \star *}$ \\
\hline Drink & $0.9 \pm 0.4$ & $0.2 \pm 0.1$ & $9.5^{\star \star}$ & $7.6 \pm 63.9$ & $2.3 \pm 2.5$ & $5.6^{*}$ \\
\hline Tailwag & $1.3 \pm 0.6$ & $0.2 \pm 0.2$ & $9.1^{\star \star}$ & --- & --- & --- \\
\hline Paw & $0.4 \pm 0.3$ & $0.0 \pm 0.0$ & $6.9^{*}$ & --- & --- & --- \\
\hline Scratch & $2.2 \pm 0.9$ & $0.9 \pm 0.5$ & & $13.5 \pm 5.5$ & $6.9 \pm 4.1$ & \\
\hline Orient observer & $0.05 \pm 0.1$ & $0.1 \pm 0.1$ & & $0.10 \pm 0.1$ & $0.2 \pm 0.4$ & \\
\hline Eat & $0.2 \pm 0.2$ & $0.3 \pm 0.3$ & & $4.1 \pm 6.3$ & $11.3 \pm 14.9$ & \\
\hline Defecate & $0.2 \pm 0.1$ & $0.1 \pm 0.1$ & & --- & --- & --- \\
\hline \multicolumn{7}{|c|}{ Any interaction with: } \\
\hline Loose substrate & $6.8 \pm 1.3$ & $4.5 \pm 1.4$ & $16.7^{\star \star \star}$ & $47.2 \pm 13.4$ & $28.9 \pm 9.7$ & $6.0^{*}$ \\
\hline Floor & $15.2 \pm 1.9$ & $12.0 \pm 2.1$ & $7.2^{*}$ & $98.4 \pm 18.7$ & $184.6 \pm 25.5$ & $19.7^{\star \star \star}$ \\
\hline (Nosing floor) & $15.3 \pm 1.9$ & $20.4 \pm 2.2$ & $7.4^{*}$ & $87.6 \pm 17.0$ & $154.6 \pm 20.5$ & $15.69^{* *}$ \\
\hline (Licking floor) & $1.4 \pm 0.5$ & $2.6 \pm 0.9$ & $11.0^{\star *}$ & $9.6 \pm 3.9$ & $29.9 \pm 13.9$ & $7.5^{\star}$ \\
\hline Arena sides & $15.9 \pm 2.2$ & $12.6 \pm 1.7$ & $4.2 \dagger$ & $82.3 \pm 14.4$ & $71.6 \pm 12.7$ & \\
\hline Neighbour pig & $0.8 \pm 0.4$ & $0.3 \pm 0.2$ & $4.2 \dagger$ & $3.8 \pm 2.8$ & $1.6 \pm 0.7$ & $4.9^{*}$ \\
\hline Drain & $5.0 \pm 1.4$ & $15.3 \pm 1.5$ & & $140.6 \pm 26.6$ & $153.5 \pm 27.6$ & \\
\hline Bars & $8.0 \pm 1.3$ & $6.2 \pm 1.1$ & & $40.3 \pm 10.0$ & $31.8 \pm 7.9$ & \\
\hline Food trough & $0.8 \pm 0.3$ & $0.6 \pm 0.3$ & & $2.4 \pm 1.1$ & $1.9 \pm 1.2$ & \\
\hline
\end{tabular}

Means are given \pm SEM. Frequencies and durations (s) were calculated over 10-min trial periods.

$\dagger P<0.06$; ${ }^{*} P<0.05 ;{ }^{* \star} P<0.01 ;{ }^{* \star *} P<0.001$. 
Table 3. Effect of treatment on the behaviour of pigs housed in substrate-enriched (SE) or substrateimpoverished $(\mathrm{SI})$ conditions in the arena novel object trial

\begin{tabular}{|c|c|c|c|c|c|c|}
\hline \multirow[b]{2}{*}{ Behaviour } & \multicolumn{2}{|c|}{ Frequency } & \multirow[b]{2}{*}{$W_{1}$} & \multicolumn{2}{|c|}{ Duration } & \multirow[b]{2}{*}{$W_{1}$} \\
\hline & SE & SI & & SE & SI & \\
\hline Change area & $5.1 \pm 0.3$ & $3.1 \pm 0.3$ & $28.8^{\star \star \star}$ & --- & --- & --- \\
\hline Stand/walk idle & $25.2 \pm 1.3$ & $3.1 \pm 0.3$ & $19.5^{\star \star \star}$ & $129.0 \pm 15.5$ & $124.6 \pm 15.6$ & \\
\hline Drink & $0.8 \pm 0.1$ & $17.4 \pm 1.3$ & $13.2^{\star \star \star}$ & $7.1 \pm 1.1$ & $1.0 \pm 1.1$ & $16.0^{\star \star \star}$ \\
\hline Scratch & $0.5 \pm 0.1$ & $0.2 \pm 0.1$ & $6.7^{\star \star}$ & $2.4 \pm 0.7$ & $0.6 \pm 0.7$ & $4.8^{*}$ \\
\hline Scamper & $0.5 \pm 0.1$ & $0.1 \pm 0.1$ & $5.2^{\star}$ & $2.0 \pm 0.6$ & $0.8 \pm 0.6$ & $4.6^{*}$ \\
\hline Tailwag & $0.9 \pm 0.3$ & $0.2 \pm 0.1$ & $5.7^{\star}$ & --- & --- & --- \\
\hline Defecate & $0.1 \pm 0.0$ & $0.1 \pm 0.3$ & $4.7^{*}$ & --- & --- & --- \\
\hline Paw & $0.2 \pm 0.1$ & $0.1 \pm 0.1$ & & --- & --- & --- \\
\hline Orient observer & $0.1 \pm 0.0$ & $0.1 \pm 0.0$ & & $0.2 \pm 0.1$ & $0.1 \pm 0.1$ & \\
\hline Eat & $0.1 \pm 0.1$ & $0.2 \pm 0.1$ & & $0.6 \pm 1.8$ & $5.4 \pm 1.8$ & \\
\hline \multicolumn{7}{|l|}{ Any interaction with: } \\
\hline Loose substrate & $5.3 \pm 0.9$ & $2.2 \pm 0.9$ & $14.4^{\star \star \star}$ & $48.8 \pm 10.2$ & $14.7 \pm 10.2$ & $14.5^{\star \star \star}$ \\
\hline Arena sides & $5.5 \pm 0.5$ & $3.2 \pm 0.5$ & $14.7^{\star \star \star}$ & $25.9 \pm 2.7$ & $15.0 \pm 2.7$ & $8.7^{\star \star}$ \\
\hline Drain & $7.5 \pm 0.9$ & $4.8 \pm 0.9$ & $5.4^{*}$ & $67.5 \pm 10.9$ & $41.3 \pm 10.9$ & \\
\hline Bars & $3.3 \pm 0.4$ & $2.1 \pm 0.4$ & $5.6^{*}$ & $16.7 \pm 2.3$ & $10.7 \pm 2.4$ & $4.2^{*}$ \\
\hline Neighbouring pig & $0.4 \pm 0.1$ & $0.2 \pm 0.1$ & $3.9^{*}$ & $1.6 \pm 0.5$ & $0.6 \pm 0.5$ & \\
\hline Floor & $7.4 \pm 1.0$ & $8.4 \pm 1.0$ & & $47.5 \pm 7.7$ & $59.5 \pm 7.8$ & \\
\hline Food trough & $0.4 \pm 0.1$ & $0.4 \pm 0.1$ & & $1.3 \pm 0.8$ & $2.2 \pm 0.8$ & \\
\hline \multicolumn{7}{|c|}{ Interaction with novel object } \\
\hline $\begin{array}{l}\text { Any interaction with novel } \\
\text { object }\end{array}$ & $10.5 \pm 0.9$ & $9.7 \pm 0.9$ & & $234.4 \pm 26.4$ & $306.6 \pm 26.6$ & \\
\hline Orient novel object & $2.1 \pm 0.5$ & $1.6 \pm 0.5$ & & $14.6 \pm 4.4$ & $14.6 \pm 4.4$ & \\
\hline Root novel object & $4.2 \pm 0.8$ & $3.1 \pm 0.8$ & & $14.5 \pm 3.2$ & $10.0 \pm 3.2$ & \\
\hline Nose novel object & $21.5 \pm 2.1$ & $24.4 \pm 2.1$ & & $101.7 \pm 11.2$ & $113.4 \pm 11.3$ & \\
\hline Chew novel object & $18.1 \pm 2.1$ & $23.9 \pm 2.1$ & & $94.5 \pm 13.6$ & $159.4 \pm 13.7$ & $4.6^{*}$ \\
\hline Lick novel object & $0.1 \pm 0.0$ & $0.03 \pm 0.0$ & & $0.2 \pm 0.1$ & $0.1 \pm 0.1$ & \\
\hline Carry novel object & $2.2 \pm 0.6$ & $2.1 \pm 0.6$ & & $9.3 \pm 2.3$ & $7.0 \pm 2.3$ & \\
\hline Shake novel object & $2.9 \pm 0.8$ & $4.3 \pm 0.8$ & & $11.2 \pm 4.5$ & $15.9 \pm 4.5$ & \\
\hline Pull novel object & $0.4 \pm 0.1$ & $0.2 \pm 0.1$ & & $1.2 \pm 0.3$ & $0.4 \pm 0.3$ & \\
\hline
\end{tabular}

Means are given \pm SEM. Frequencies and durations (s) were calculated over 10-min trial periods.

${ }^{\star} P<0.05 ;{ }^{*} P<0.01 ;{ }^{\star * \star} P<0.001$.

This hypothesis accords with a model of stereotyped behaviour formulated by Lawrence \& Terlouw (1993) and Rushen et al. (1993), who suggested that the static, unvarying character of restrictive environments affects the organization of behaviour by a process they called 'environmental channelling'. Such channelling, they argued, may help to explain the strikingly rigid and fixed form of stereotyped behaviours. 'We know that under relatively unrestricted conditions, such as the Edinburgh Pig Park, foraging by pigs involves a variable and complex set of behaviours. Under the more restricted conditions of a stall, this behaviour can only be expressed against a few static substrates such as chains and bars. 
Potentially complex behaviour is therefore channelled and simplified by the unvarying characteristics of the environment' (Rushen et al. 1993, page 50).

In the light of this model, the reduction in relative behavioural diversity observed both in the home pen and in the test situations could be regarded as a preliminary stage of environmental channelling, and could signal that pigs are at risk of developing more stereotyped forms of behaviour. The gradual increase in vacuum chewing shown by SI animals in the home pen (Haskell et al. 1996) supports this interpretation. If pigs are deprived of complex foraging opportunities, vacuum chewing tends to become stereotyped (Bergeron \& Gonyou 1997). Similarly, the persistently high levels of chewing the novel object by SI pigs may be an early sign of the gradual fixation of appetitive and ingestive behaviours (cf. Hughes \& Duncan 1988).

A similar interpretation can be derived from dynamic systems theory, a theoretical approach that has provided a fruitful new perspective on behavioural learning and development in young human infants (Fogel \& Thelen 1987; Thelen 1995). This theory conceives of behavioural diversification as an organism's ability to create unstable states of organization in response to novel stimulation, allowing the organism to transcend constraints of learned patterns of behaviour and achieve greater flexibility of movement. Stable states of organization, on the other hand, facilitate the practice and consolidation of acquired behavioural skills, and are characterized by persistence of movement rather than spontaneous variability. Normal behavioural development from this perspective can be conceived as a sequence of stable and unstable dynamic landscapes (Thelen 1995). In this light, the tendency of SI pigs to focus behaviour on particular substrates at the expense of mobility and diversity may indicate an inappropriate persistence of stable state landscape parameters under novel conditions, which in the long run could disturb normal behavioural development.

Our results also present some problems to such an interpretation. First, if indeed SI pigs were subject to a process of gradual behavioural fixation, one would expect the observed differences between SI and SE animals to increase over the 5 months of the experiment. This, however, was the case only with chewing the novel object during the arena novel object test, where SI pigs showed a strong increase in levels of chewing and SE pigs did not. In all other cases, including relative diversity index, $R$, treatment effects were present from the first observations and did not increase further. This may mean that the treatments were not dissimilar enough for differences in behaviour to increase over time. The observed effects might also have been greater if the trial had begun at an earlier age. Alternatively, these results may indicate that environmental channelling does not occur as a smooth and gradual process but involves more sudden transformations. Further research over a more prolonged period is needed to differentiate between these explanations.

Second, the proposed interpretation apparently contradicts Mendl et al. (1997). These authors used the same SI and SE pigs as in the present study and, at the end of the 5-month experimental period, subjected them to a series of T-maze spatial discrimination tasks motivated by a food reward. The hypothesis tested was that SI pigs would show a more fixed, unvarying response to these tasks than SE pigs, for example by being slower to acquire a reversal task. However, the results did not support this hypothesis; if anything it was the SE pigs that tended to respond with routine-like behaviour. These tests, however, focused on specific measures of the ability to inhibit and alter previous response patterns in a food-motivated task, and used animals that were 'forced' to explore a maze away from their home pens. As such, they provide information about the development of routine-like behaviour, but are quite different to the 'free', voluntary nature of our novel object tests (see Introduction). The presence of food rewards can also change the way in which animals behave under novel conditions (Harlow et al. 1950; Mendl et al. 1997). Comparison of our results with those of the T-maze tests is therefore not straightforward (cf. Mendl \& Deag 1995). 
Table 4. Effect of treatment on the behaviour of pigs housed in substrate-enriched (SE) or substrateimpoverished (SI) conditions in the home pen novel object test

\begin{tabular}{|c|c|c|c|c|c|c|}
\hline \multirow[b]{2}{*}{ Behaviour } & \multicolumn{2}{|c|}{ Frequency } & \multirow[b]{2}{*}{$W_{1}$} & \multicolumn{2}{|c|}{ Duration } & \multirow[b]{2}{*}{$W_{1}$} \\
\hline & SE & SI & & SE & SI & \\
\hline Drink & $1.1 \pm 0.1$ & $0.4 \pm 0.14$ & $16.5^{\star \star \star}$ & $12.2 \pm 2.2$ & $4.5 \pm 2.2$ & $7.7^{\star \star}$ \\
\hline Stand/walk idle & $15.8 \pm 1.3$ & $12.1 \pm 1.33$ & $4.4^{*}$ & $78.0 \pm 10.7$ & $57.1 \pm 10.7$ & $3.7 \dagger$ \\
\hline Orient observer & $0.1 \pm 0.0$ & $0.0 \pm 0.03$ & $4.0^{*}$ & $0.3 \pm 0.1$ & $0.1 \pm 0.1$ & \\
\hline Eat & $0.3 \pm 0.2$ & $0.3 \pm 0.15$ & & $15.6 \pm 8.1$ & $10.3 \pm 8.1$ & \\
\hline Scamper & $0.1 \pm 0.1$ & $0.1 \pm 0.05$ & & $0.3 \pm 0.1$ & $0.2 \pm 0.1$ & \\
\hline Tailwag & $0.4 \pm 0.2$ & $0.4 \pm 0.15$ & & --- & --- & --- \\
\hline Defecate & $0.0 \pm 0.0$ & $0.0 \pm 0.01$ & & --- & --- & --- \\
\hline Paw & $1.7 \pm 0.5$ & $0.8 \pm 0.45$ & & --- & --- & --- \\
\hline Scratch & $0.1 \pm 0.0$ & $0.1 \pm 0.03$ & & $0.1 \pm 0.1$ & $0.1 \pm 0.1$ & \\
\hline \multicolumn{7}{|l|}{ Any interaction with: } \\
\hline Floor & $0.2 \pm 0.5$ & $3.4 \pm 0.53$ & $38.8^{\star \star \star}$ & $1.0 \pm 4.1$ & $21.4 \pm 4.1$ & $29.6^{\star \star \star}$ \\
\hline Loose substrate & $6.3 \pm 0.5$ & $2.1 \pm 0.51$ & $41.1^{\star \star \star}$ & $74.4 \pm 7.1$ & $11.6 \pm 7.1$ & $49.8^{\star \star \star}$ \\
\hline Neighbouring pig & $0.7 \pm 0.1$ & $0.5 \pm 0.11$ & $6.8^{\star \star}$ & $2.3 \pm 0.6$ & $2.2 \pm 0.6$ & \\
\hline Bars & $2.5 \pm 0.4$ & $1.7 \pm 0.36$ & $4.5^{\star}$ & $9.2 \pm 2.1$ & $6.3 \pm 2.1$ & \\
\hline Food trough & $0.5 \pm 0.1$ & $0.3 \pm 0.13$ & & $1.9 \pm 0.6$ & $1.5 \pm 0.6$ & \\
\hline \multicolumn{7}{|c|}{ Interaction with novel object } \\
\hline $\begin{array}{l}\text { Any interaction with novel } \\
\text { object }\end{array}$ & $12.4 \pm 1.0$ & $11.9 \pm 1.00$ & & $404.7 \pm 21.0$ & $483.6 \pm 21.0$ & $7.1^{\star \star}$ \\
\hline Root novel object & $16.5 \pm 1.7$ & $12.2 \pm 1.66$ & $3.8^{*}$ & $59.8 \pm 6.9$ & $40.9 \pm 6.9$ & $4.2^{*}$ \\
\hline Nose novel object & $35.9 \pm 1.8$ & $40.9 \pm 1.79$ & $4.3^{*}$ & $174.1 \pm 10.2$ & $194.6 \pm 10.2$ & \\
\hline Chew novel object & $30.6 \pm 2.3$ & $38.3 \pm 2.25$ & $6.2^{\star}$ & $155.8 \pm 14.9$ & $232.5 \pm 14.9$ & $14.2^{\star \star \star}$ \\
\hline Orient novel object & $0.0 \pm 0.3$ & $0.2 \pm 0.07$ & $3.4 \dagger$ & $0.1 \pm 0.3$ & $1.1 \pm 0.3$ & $4.7^{*}$ \\
\hline Lick novel object & $0.9 \pm 0.1$ & $0.7 \pm 0.34$ & & $3.7 \pm 1.2$ & $2.3 \pm 1.2$ & \\
\hline Carry novel object & $1.1 \pm 0.4$ & $0.7 \pm 0.38$ & & $2.9 \pm 1.1$ & $2.2 \pm 1.1$ & \\
\hline Shake novel object & $1.7 \pm 0.8$ & $2.7 \pm 0.76$ & & $4.9 \pm 3.0$ & $9.1 \pm 3.0$ & \\
\hline Pull novel object & $0.3 \pm 0.2$ & $0.4 \pm 0.17$ & & $0.9 \pm 0.5$ & $1.2 \pm 0.5$ & \\
\hline
\end{tabular}

Means are given \pm SEM. Frequencies and durations (s) were calculated over 10-min trial periods.

$\dagger P<0.06^{\star} P<0.05 ;{ }^{\star \star} P<0.01 ;{ }^{* \star *} P<0.001$.

In conclusion, our results show that the reduced behavioural diversity of animals housed in substrateimpoverished conditions (Haskell et al. 1996) persisted in two novel object tests. Thus they support the hypothesis that substrate-impoverished housing conditions structurally affect the propensity of pigs to explore and interact with their environment. This study indicates that 'free exploration' experimental conditions are an effective tool in the investigation of dynamic aspects of behavioural organization, and as such should stimulate further research.

\section{Acknowledgments}

We thank Lesley Deans and Hans Erhard for their assistance with the observations, Joan Chirnside and Kirsty Mclean for taking care of the animals, David Hitchcock and Elizabeth Austin of BIOSS for statistical advice and two anonymous referees for helpful comments on the manuscript. This research was funded 
by the BBSRC of the UK, the Scottish Office Agriculture, Environment and Fisheries Department, and the Netherlands Organization for Scientific Research. We are grateful to CABI Publishing for permission to quote from Rushen et al. (1993).

\section{References}

Adams, D. C. \& Anthony, C. D. 1996. Using randomization techniques to analyse behavioural data. Animal Behaviour, 51, 733-738.

Belzung, C. \& Le Pape, G. 1994. Comparison of different behavioral test situations used in psychopharmacology for measurement of anxiety. Physiology and Behavior, 56, 623-628.

Bergeron, R. \& Gonyou, H. W. 1997. Effects of increasing energy intake and foraging behaviours on the development of stereotypies in pregnant sows. Applied Animal Behaviour Science, 53, 259-270.

Boissy, A. \& Bouissou, M.-F. 1995. Assessment of individual differences in behavioural reactions of heifers exposed to various fear-eliciting situations. Applied Animal Behaviour Science, 46, 17-31.

Dantzer, R. 1986. Behavioral, physiological and functional aspects of stereotyped behaviour: a review and a re-interpretation. Journal of Animal Science, 62, 1776-1786.

Deag, J. M. 1993. Keytime: a program system for recording and analysing behavioural data. Copyright John M. Deag 1987-1993, 10 Fletcher Grove, Penicuik, Midlothian, U.K.

Einon, D., Morgan, M. J. \& Kibbler, C. C. 1978. Brief periods of socialization and later behaviour in the rat. Developmental Psychology, 11, 213-225.

Fogel, A. \& Thelen, E. 1987. Development of early expressive and communicative action: reinterpreting the evidence from a dynamic systems perspective. Developmental Psychology, 23, 747-761.

Gunn, D. \& Morton, D.B. 1995. Inventory of the behaviour of New Zealand White rabbits in laboratory cages. Applied Animal Behaviour Science, 45, 277-292.

Harlow, H. F., Harlow, M. K. \& Meyer, D. R. 1950. Learning motivated by a manipulation drive. Journal of Experimental Psychology, 40, 228-234.

Haskell, M., Wemelsfelder, F., Mendl, M., Calvert, S. \& Lawrence, A. B. 1996. The effect of substrateenriched and susbstrate-impoverished housing environments on the diversity of behaviour in pigs. Behaviour, 133, 741-761.

Hughes, B. O. \& Duncan, I. J. H. 1988. The notion of ethological 'need', models of motivation and animal welfare. Animal Behaviour, 36, 1696-1707.

Lawrence, A. B. \& Terlouw, E. M. C. 1993. A review of behavioral factors involved in the development and continued performance of stereotypic behaviors in pigs. Journal of Animal Science, 71, 2815-2825.

Lawes Agricultural Trust 1993. Genstat 5 Release 3 Reference Manual. Oxford: Oxford University Press.

Losey, G. S. 1978. Information theory and communication. In: Quantitative Ethology (Ed. By P. W. Colgan), pp. 43-78. New York: J. Wiley.

McKinnon, A. J., Edwards, S. A., Stephens, D. B. \& Walters, D. E. 1989. Behaviour of groups of weaner pigs in three different housing systems. British Veterinary Journal, 145, 367-372.

Mason, G. J. 1991. Stereotypies: a critical review. Animal Behaviour, 41, 1015-1037.

Meijsser, F. M., Kersten, A. M. P., Wiepkema, P. R. \& Metz, J. H. M. 1989. An analysis of the open-field performance of sub-adult rabbits. Applied Animal Behaviour Science, 24, 147-155.

Mendl, M. \& Deag, J. M. 1995. How useful are the concepts of alternative strategy and coping strategy in applied studies of social behaviour? Applied Animal Behaviour Science, 44, 119-137.

Mendl, M., Erhard, H. W., Haskell, M., Wemelsfelder, F. \& Lawrence, A. B. 1997. Experience in substrateenriched and substrate-impoverished environments affects behaviour of pigs in a T-MAZE task. Behaviour, 134, 643-659.

Morgan, M. J. 1973. Effects of post-weaning environment on learning in the rat. Animal Behaviour, 21, 429-442. 
Page, S. \& Neuringer, A. 1985. Variability is an operant. Journal of Experimental Psychology: Animal Behavior Processes, 11, 429-452.

de Passillé, A. M., Rushen, J. \& Martin, F. 1995. Interpreting the behaviour of calves in an open-field test: a factor analysis. Applied Animal Behaviour Science, 45, 201-213.

Patterson, H. D. \& Thompson, R. 1971. Recovery of inter-block information when block sizes are unequal. Biometrika, 58, 545-554.

Pearce, G. P. \& Paterson, A. M. 1993. The effect of space restriction and provision of toys during rearing on the behaviour, productivity and physiology of male pigs. Applied Animal Behaviour Science, $36,11-28$.

Petersen, V., Simonsen, H. B. \& Lawson, L. G. 1995. The effect of environmental stimulation on the development of behaviour in pigs. Applied Animal Behaviour Science, 45, 215-224.

Prior, H. \& Sachzer, N. 1994. Effects of enriched housing environment on the behaviour of young female mice in four exploratory tasks. Journal of Experimental Animal Science, 37, 57-68.

Renner, M. J. 1987. Experience-dependent changes in exploratory behavior in the adult rat (Rattus norvegicus): overall activity level and interactions with objects. Journal of Comparative Psychology, 101, 94-100.

Renner, M. J. \& Rosenzweig, M. R. 1986. Object interactions in juvenile rats (Rattus norvegicus): effects of different experiential histories. Journal of Comparative Physiology and Psychology, 100, 229236.

Rushen, J., Lawrence, A. B. \& Terlouw, E. M. C. 1993. The motivational basis of stereotypies. In: Stereotypic Animal Behaviour: Fundamentals and Applications to Welfare (Ed. by A. B. Lawrence \& J. Rushen), pp. 41-65. Wallingford: CAB International.

Stolba, A., Baker, N. \& Wood-Gush, D. G. M. 1983. The characterization of stereotyped behaviour in stalled sows by informational redundancy. Behaviour, 87, 157-182.

Thelen, E. 1995. Motor development: a new synthesis. American Psychologist, 50, 79-95.

Treit, D. 1985. Animal models for the study of anti-anxiety agents: a review. Neuroscience and Biobehavioral Reviews, 9, 203-222.

Welker, W. I. 1957. 'Free' versus 'forced' exploration of a novel situation by rats. Psychological Reports, 3, 95-108.

Wemelsfelder, F. 1993. The concept of animal boredom and its relationship to stereotyped behaviour. In: Stereotypic Animal Behaviour: Fundamentals and Applications to Welfare (Ed. by A. B. Lawrence \& J. Rushen), pp. 65-97. Wallingford: CAB International.

Wood-Gush, D. G. M. \& Vestergaard, K. 1989. Exploratory behavior and the welfare of intensively kept animals. Journal of Agricultural Ethics, 2, 161-169.

Wood-Gush, D. G. M. \& Vestergaard, K. 1991. The seeking of novelty and its relation to play. Animal Behaviour, 42, 599-606.

Wood-Gush, D. G. M., Vestergaard, K. \& Petersen, H. V. 1990. The significance of motivation and environment in the development of exploration in pigs. Biology of Behaviour, 15, 39-52. 